\title{
BMJ Open Is subjectively perceived treatment urgency of patients in emergency departments associated with self-reported health literacy and the willingness to use the GP as coordinator of treatment? Results from the multicentre, cross- sectional, observational study PiNo Bund
}

\author{
Ingmar Schäfer (D , , ${ }^{1}$ Agata Menzel, ${ }^{1}$ Jan Hendrik Oltrogge, ${ }^{1}$ Anna Slagman, ${ }^{2}$ \\ Martin Möckel (D) ,2 Dagmar Lühmann, ${ }^{1}$ Martin Scherer ${ }^{1}$
}

To cite: Schäfer I, Menzel A Oltrogge $\mathrm{JH}$, et al. Is subjectively perceived treatment urgency of patients in emergency departments associated with self-reported health literacy and the willingness to use the GP as coordinator of treatment? Results from the multicentre, cross-sectional, observational study PiNo Bund. BMJ Open 2021;11:e053110. doi:10.1136/ bmjopen-2021-053110

- Prepublication history for this paper is available online. To view these files, please visit the journal online (http://dx.doi org/10.1136/bmjopen-2021053110).

Received 05 May 2021 Accepted 31 October 2021

Check for updates

(C) Author(s) (or their employer(s)) 2021. Re-use permitted under CC BY-NC. No commercial re-use. See rights and permissions. Published by BMJ.

For numbered affiliations see end of article.

Correspondence to Dr Ingmar Schäfer; in.schaefer@uke.de

\section{ABSTRACT}

Objectives Aim of this study was to analyse if subjectively perceived treatment urgency of patients in emergency departments is associated with self-reported health literacy and the willingness to use the general practitioner (GP) as coordinator of treatment.

Design A multicentre, cross-sectional, observational study.

Setting Emergency departments in five hospitals. Each hospital was visited 14 times representing two 8-hour shifts on each day of the week. Calendar dates were randomly assigned.

Participants All patients of legal age registered at the emergency department or hospital reception desk. Exclusion criteria included immediate or very urgent need of treatment, high level of symptom burden and severe functional impairments in terms of hearing, vision and speech. We conducted standardised personal interviews. Additionally, clinical data were extracted from patient records.

Primary and secondary outcome measures Our target variable was subjectively perceived treatment urgency. Predictor variables included age, sex, education, healthrelated quality of life (EuroQol Five-Dimension Scale, value set UK), anxiety and depression (Hospital Anxiety and Depression Scale), somatic symptoms (Patient Health Questionnaire, 15 items version), self-reported health literacy (European Health Literacy Questionnaire, 16 questions version) and the commitment to the GP (Fragebogen zur Intensität der Hausarztbindung, 'F-HaBi'). Data were analysed by multilevel, multivariable linear regression adjusted for random effects at the hospital level.

Results Our sample comprised 276 patients with a mean age of 50.1 years and $51.8 \%$ women. A low treatment urgency (defined as $0-5$ points on a Numerical Rating Scale) was reported by 111 patients (40.2\%). In the final model, lower subjective treatment urgency was associated with male sex $(\beta=0.84 ; 95 \% \mathrm{Cl} 0.11 / 1.57$,
Strengths and limitations of this study

- We made a careful selection of included hospitals based on an analysis of the outpatient and inpatient care situation throughout Germany.

- The interviewers received multiple training sessions before the interviews started and were supervised throughout the entire survey period.

- The statistical methods provided additional strength by considering potential confounders and the cluster structure of the dataset.

- We did not recruit hospitals from districts with a low rate of ambulatory care-sensitive cases in emergency departments, and mostly rural regions have been selected for our study.

- We did not conduct a sample size calculation and we might, therefore, have missed significant predictors of our target variable due to limited statistical power.

$\mathrm{p}=0.024)$, higher health-related quality of life $(-2.27$ to $-3.39 /-1.15, p<0.001)$, lower somatic symptoms score $(0.09,0.004 / 0.17, p=0.040)$, higher anxiety score $(-0.13$ to $-0.24 /-0.01, p=0.027)$ and lower commitment to the GP $(0.08,0.01 / 0.14, p=0.029)$.

Conclusions A lower level of subjectively perceived treatment urgency was predicted by a lower willingness to use the GP as coordinator of treatment. Self-reported health literacy did not predict the patients' urgency rating.

\section{BACKGROUND}

Patient numbers in emergency departments are rising since many years and this increase cannot be explained by population growth alone. ${ }^{1-3}$ Emergency department crowding is challenging for the healthcare system in many countries worldwide. For patients, 
overcrowded emergency departments are associated with delays in the assessment of symptoms and delivery of care, with exposure to error, increased length of stay and-under certain circumstances-increased patient mortality. Studies also have identified various negative effects on the hospital staff such as increased stress, exposure to violence and non-adherence to clinical guidelines. ${ }^{3-5}$

According to the specialist societies of emergency medicine in German-speaking countries, medical emergencies can be defined as changes in health for which the patients themselves or third parties deem immediate medical and nursing care to be required. ${ }^{6}$ Even under this permissive definition, a substantial proportion of emergency department visits cannot be classified as medical emergency, because they are conducted by self-referring patients who perceive the treatment urgency of their health problems as low. ${ }^{7}$

In various other studies, we have explored the reasons for encounter in emergency departments before. ${ }^{7-9}$ In these studies, special attention has been paid on the role of the primary care setting, which is upstream of the emergency departments. ${ }^{10}$ Patient preferences and lack of knowledge regarding other treatment options were frequent reasons for visiting emergency departments. For example, in the 'PiNo Nord' study, $67.4 \%$ of the patients did not know the emergency house call services of the Associations of Statutory Health Insurance Physicians and $55.2 \%$ did not know their emergency practices. And $19.1 \%$ preferred treatment in the emergency department to outpatient treatment due to the diagnostic and treatment facilities of the hospital. ${ }^{7}$

For this reason, the patients' commitment to a general practitioner (GP) and their health literacy might influence the decision to use emergency departments for conditions with low subjective treatment urgency. The so-called 'commitment to a general practitioner' is a new concept, investigating to what extent patients voluntarily use their GP's gatekeeping role or whether they move independently in the healthcare system instead. According to this concept, the commitment to a GP is strong if patients (1) consult their GP first in all healthcare issues, (2) let their GP to coordinate all of their healthcare issues and (3) have a high amount of trust to their GP. ${ }^{11}$ Among other factors, a higher willingness to use the GP as coordinator of treatment is associated with male sex, a lower socioeconomic status and a higher number of chronic diseases. ${ }^{11}$ The commitment to a GP has to be distinguished from 'health literacy', which describes the ability of an individual to access and understand health information in order to take decisions concerning healthcare, disease prevention and health promotion. $^{12}$

The aim of this study was to analyse if subjectively perceived treatment urgency of patients in emergency departments is associated with their self-reported health literacy and their willingness to use the GP as coordinator of treatment.

\section{METHODS}

\section{Design and setting}

PiNo Bund ('Patienten in der Notaufnahme von Kliniken in der Bundesrepublik Deutschland'; patients in the emergency departments of hospitals in Germany) is a multicentre, cross-sectional, observational study conducted in five hospitals. The selection of eligible hospitals was guided by an unpublished cluster analysis of the Central Research Institute of Ambulatory Health Care in Germany. ${ }^{13}$ This analysis was based on (1) the number of office-based statutory health insurance physicians (physician density), (2) the number of beds in hospitals for acute cases (bed density), (3) the total number of ambulatory care-sensitive cases ${ }^{14}$ and (4) the number of ambulatory care-sensitive cases in emergency departments (ACS density) per 100000 inhabitants in each specific administration district in Germany.

For our study, we selected hospitals from districts in which the ACS density was medium to very high, which applied to three out of five clusters identified in the analysis (cf. figure 1). The selected hospitals were located in the German federal states of Brandenburg, Lower Saxony, North Rhine-Westphalia and Saxony-Anhalt, and included the following cities and districts:

- Dessau-Roßlau (80 000 inhabitants, rural district with signs of agglomeration).

- Lingen/Ems (55000 inhabitants) in the county Emsland (sparsely populated rural district).

- Frankfurt/Oder (58 000 inhabitants, sparsely populated rural district).

- Soest (48 000 inhabitants, urbanised district).

- Wernigerode (33000 inhabitants) in the county Harz (sparsely populated rural district ${ }^{15}$ ).

Each hospital was visited twice before the start of the project in order to (1) adapt the procedures of patient recruitment and data collection to the local situation and (2) train the project staff in the work process. Additionally, the staff was trained in two webinars before project start and continuously monitored by telephone during data collection.

\section{Patient and public involvement}

There was no patient and public involvement in the design, conduct and reporting of our research.

\section{Patient population, recruitment and data collection}

Data collection took place from 21 January 2019 to 14 April 2019. During this time, each hospital was visited 14 times representing two 8-hour shifts (6:00-14:00 and 14:00-22:00) on each day of the week (Monday-Sunday). The specific calendar dates for each visit were randomly assigned within the observation period. The decision to exclude night shifts was based on the low patient numbers observed between 22:00 and 6:00 in our prior research with similar design. ${ }^{7}$

On each working day, one project member documented all patients who attended the emergency department and checked them for inclusion and exclusion criteria. We 


\begin{tabular}{|c|c|c|}
\hline \multicolumn{3}{|c|}{ Characteristics of districts } \\
\hline Physician density & Bed density & ACS density \\
\hline low & very low & very low \\
\hline very low & low & medium \\
\hline low & medium & very high \\
\hline high & very high & high \\
\hline high & medium & low \\
\hline
\end{tabular}

Selected locations: Cities, districts, federal states

(1) Lingen/Ems, Emsland, Lower Saxony

Soest, Soest, North Rhine-Westphalia

Wernigerode, Harz, Saxony-Anhalt

Dessau-Roßlau, Dessau-Roßlau, Saxony-Anhalt

Frankfurt/Oder, Frankfurt/Oder, Brandenburg

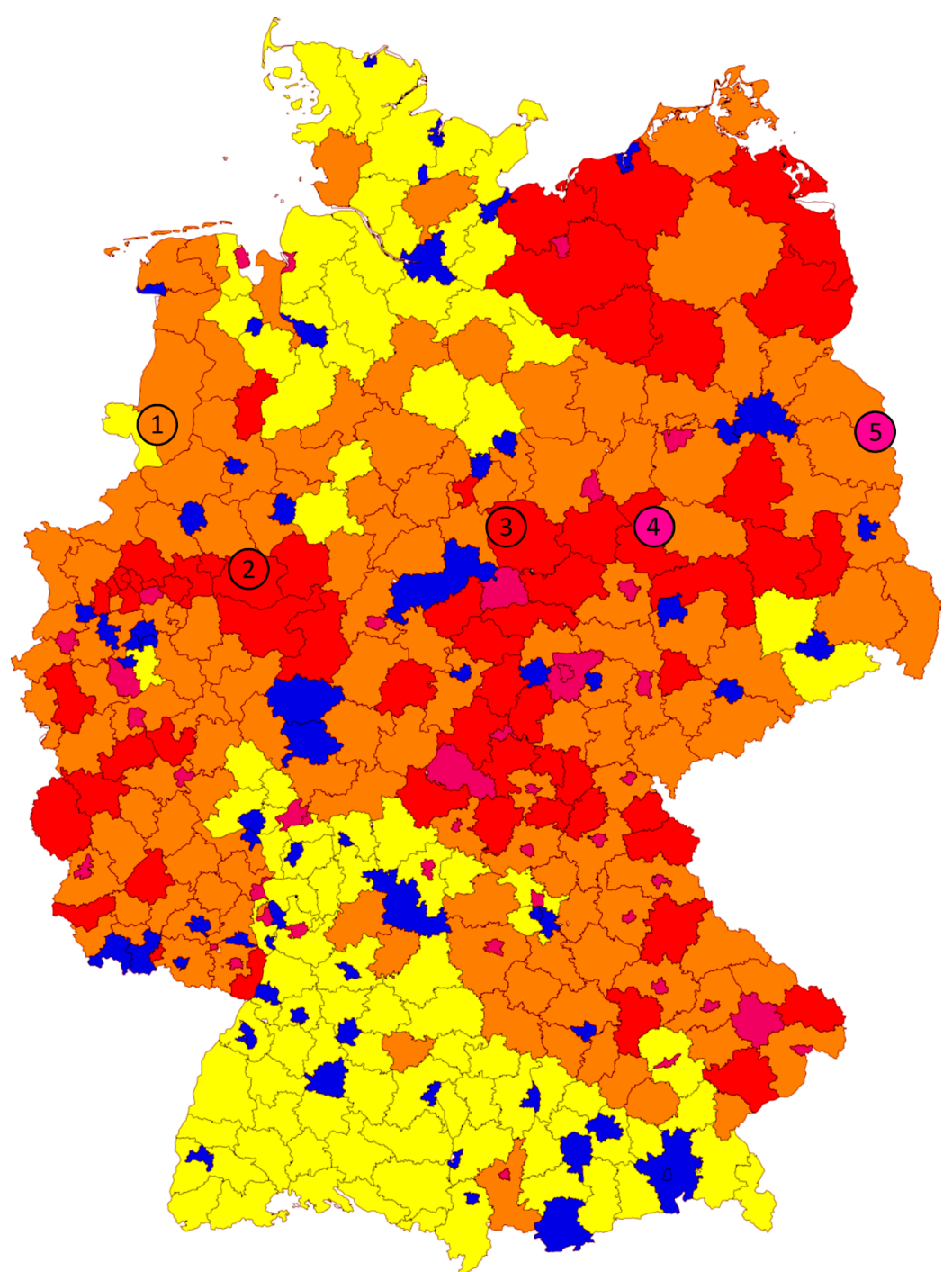

Figure 1 Characteristics of districts and selected locations. Copyright holder: GeoBasis-DE/German Federal Agency for Cartography and Geodesy (BKG) 2017 (adjusted). Contains results from an unpublished analysis of Erhart et al. ${ }^{13}$ ACS, ambulatory care-sensitive cases in emergency departments.

included all patients of legal age (ie, 18 years or older) who had been registered at the emergency department or hospital reception desk. We excluded patients with immediate or very urgent need of treatment (based on triage level or-if not available-opinion of the hospital staff), high level of symptom burden or severe functional impairments in terms of hearing, vision and speech (each assessed by hospital staff). We also excluded patients who had been treated without waiting period or were directly referred to another department, patients without capacity to consent, patients with whom verbal communication in German was not possible and patients who had received a regular (non-emergency) hospital admission or had to be isolated because of a (presumed) contagious disease.

Eligible patients were asked for informed consent. If consent was given, the patient was personally interviewed by a second project member using a standardised questionnaire. Patient recruitment and personal interviews were conducted by employees of USUMA ('Unabhängiger Service für Umfragen, Methoden und Analysen'), an independent market and social research institute. After the interviews were finished, clinical data were extracted from the hospitals' patient records.

\section{Target and predictor variables}

Target variable of our analyses was the subjectively perceived treatment urgency. As opposed to a definition based on the level of subjective illness burden, in our study, treatment urgency was defined by the degree to which the patients perceived their health problem as life threatening. In the interviews, the patients estimated how urgently they needed treatment by using a Numerical Rating Scale from 0 (indicating 'no urgent need for treatment') to 10 (indicating 'very urgent, life threatening'). Additionally, the triage level indicating the treatment urgency assessed by the hospitals' medical and nursing staff and the medical diagnoses documented at the emergency departments were extracted from the hospitals' patient records. Retrospectively, the diagnoses were coded in the International Classification of Primary 
Care, Second Revision, ${ }^{16}$ by the project staff, which facilitates grouping by organ systems (eg, 'respiratory system' or 'psychological disorders') and by diagnosis type (ie, 'symptoms/complaints', 'infections', 'injuries', 'congenital anomalies', 'neoplasms' and 'other diagnoses'). The Manchester triage system, ${ }^{17}$ which was used in all participating hospitals, describes the clinical rating of the treatment urgency in five categories ('immediate', 'very urgent', 'urgent', 'standard' and 'non-urgent').

In the interviews, age, sex and educational level were documented as sociodemographic variables. For determining the educational level, the patients' general and vocational education were classified into three groups, according to the Comparative Analysis of Social Mobility in Industrial Nations classification system, and using 'low' for inadequately completed general education, general elementary education or basic vocational qualification; 'medium' for secondary school certificate or A level equivalent; and 'high educational level' for higher or lower tertiary education. ${ }^{18}$

The other predictor variables were assessed during the interviews and included health status, self-reported health literacy and commitment to the GP. The health status was operationalised by limitations in the health-related quality of life, and the level of anxiousness, depressiveness and somatic symptoms. Health-related quality of life was measured by the EuroQol Five-Dimension Scale (EQ5D) comprising the domains mobility, self-care, usual activities, pain or discomfort and anxiety or depression. ${ }^{19}$ An EQ-5D summary score was calculated using the value set UK. It indicates the value 1.000 for full health, which is reduced by a severity-related subtrahend between -0.081 and -0.350 if any limitations occur and up to five additional subtrahends between -0.036 and -0.386 depending on the severity of limitations in the five dimensions. ${ }^{20}$ Thus, lower scores in the EQ-5D indicate a higher level of impairment in quality of life.

The interviews also included the Hospital Anxiety and Depression Scale (HADS), ${ }^{21}$ which is specifically designed for the hospital setting. HADS consists of two subscales with seven items each, describing the severity of anxiousness and depressiveness. All items are assessed on a fourpoint Likert scale. The scores in both subscales range from 0 to 21. Additionally, we used the somatic symptoms subscale of the Patient Health Questionnaire, 15 items version (PHQ-15), ${ }^{22}$ which comprises a severity rating of 15 somatic symptoms. The PHQ-15 is summarised in a score ranging from 0 to 30 . Higher scores in HADS and PHQ-15 stand for a higher level of anxiousness, depressiveness and somatic symptoms, respectively.

Self-reported health literacy was assessed using the short form of the European Health Literacy Questionnaire, 16 questions version (HLS-EU-Q16). It includes 16 questions focusing on the four health literacy dimensions accessing, understanding, appraising and applying information to take decisions concerning healthcare (7 questions), disease prevention (5 questions) and health promotion (4 questions) ${ }^{23}$ Items were scaled on a four-point Likert scale and dichotomised for analysis grouping 'fairly easy' and 'very easy' to the value of 1 and 'fairly difficult' and 'very difficult' to the value of 0 . Thus, the HLS-EU-Q16 summary score ranges from 0 to 16 points with lower scores implying a worse health literacy.

Commitment to the GP was collected using the Questionnaire on Intensity of the Commitment to the GP ('Fragebogen zur Intensität der Hausarztbindung (F-HaBi)'). F-HaBi is a patient questionnaire examining the attitudes and behaviour regarding utilisation of GPs and medical specialists, and constitutes a summary score between 0 point and 24 points. Higher scores indicate that the patient more likely recognises and uses the GP as coordinator. Lower scores indicate that the patient prefers to move independently in the healthcare system. ${ }^{11}$ The F-HaBi also contains an item regarding if the patient has a regular GP.

\section{Statistical analyses}

A non-responder analysis was conducted using $\chi^{2}$ tests and t-tests to assess differences in age and sex between study participants and eligible patients who did not participate in our study. Data of study participants were analysed in two steps. In the first step, data analysis was carried out using descriptive statistics. $\chi^{2}$ tests and t-tests were performed to describe the differences in sociodemographic data, health status, self-reported health literacy and commitment to the GP between patients with high and low subjective treatment urgency. For these analyses, subjective treatment urgency was dichotomised. Low subjective treatment urgency was assigned if it ranged between 0 point and 5 points and high subjective treatment urgency was assigned if it ranged between 6 points and 10 points. This cut-off point was defined considering that patients who could not or would not decide about their treatment urgency and, therefore, chose the middle category best fit into the 'non-urgent' group. ${ }^{24}$ Additionally, the association between subjective treatment urgency and the triage level by the hospital staff was analysed by an ordinal logistic regression analysis.

In the second step, multilevel, multivariable linear regression models adjusted for random effects at the hospital level were conducted to analyse the association between predictor variables and subjective treatment urgency (dependent variable). These statistical analyses were used to test three hypotheses: (1) subjective treatment urgency depends in part on the patients' age, sex and/or educational level; (2) the health-related quality of life and/or patient perceptions of somatic symptoms, anxiousness and/or depressiveness are also associated with the patients' perceptions of treatment urgency and to some extent these variables explain the relationship between subjective treatment urgency and sociodemographic data and (3) patients with worse health literacy and/or less willingness to use GPs as coordinators of the treatment are more likely to visit emergency departments with health problems that are perceived by themselves as non-urgent and, therefore, lower subjective treatment 


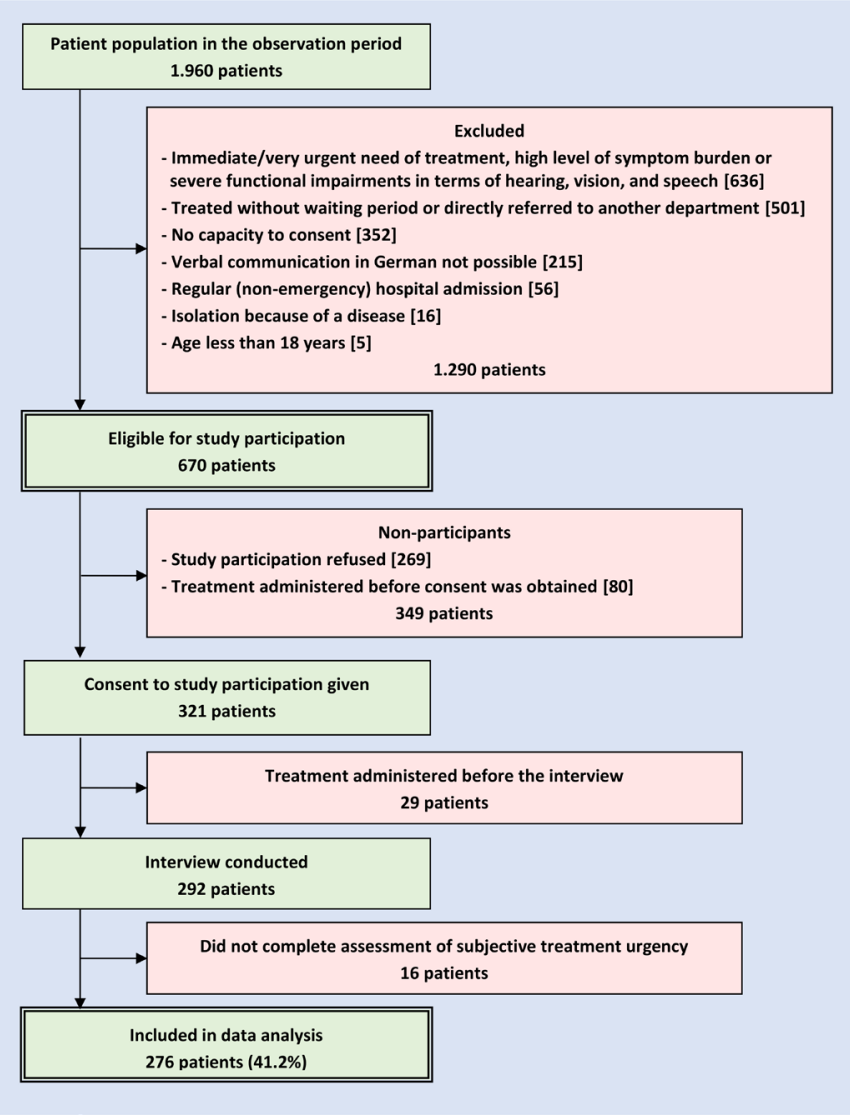

Figure 2 Recruitment process.

urgency is associated with lower health literacy and/or lower commitment to the GP. These associations are to some extent independent of sociodemographic data and health status.

The potential predictors of the treatment urgency were, therefore, subsequently included in three models. Model 1 comprised sociodemographic data as independent variables, model 2 comprised the variables from model 1 plus health status and model 3 comprised the variables from model 2 plus self-reported health literacy and commitment to the GP. In these analyses, treatment urgency was introduced as continuous variable and without transforming the collected data. A possible improvement in the model fit resulting from the inclusion of additional variables compared with the next variable-reduced nested model was determined by the likelihood ratio test. Results from inferential statistics were reported as $\beta$-coefficients with $95 \%$ CIs. An alpha level of $5 \%(p \leq 0.05)$ was defined as statistically significant. All statistical analyses were performed using Stata V.15.1.

\section{RESULTS}

\section{Recruitment}

The patient recruitment process is shown in figure 2. During our visits in the participating hospitals, 1.960 patients registered at the emergency department or hospital reception desk. A total of 1290 patients was not eligible for the study, mostly due to treatment urgency, symptom burden or functional impairment, or because they were treated without waiting or directly referred. Of the 670 eligible patients, $269(40.1 \%)$ refused study participation and another 80 patients were treated before informed consent could be obtained. We could include 321 patients into the study, but 29 interviews could not be conducted, because the treatment started before or during the survey. In the end, 292 interviews could be completed. Afterwards, we excluded another 16 patients, because of missing values in the subjectively perceived treatment urgency. Our final sample comprised 276 patients, which corresponds to a response rate of $41.2 \%$. There were no statistically significant differences between study participants and eligible non-participants regarding age ( 50.1 years vs 50.7 years, $\mathrm{p}=0.845$ ) and sex ( $51.8 \%$ women vs $53.5 \%$ women, $\mathrm{p}=0.667$ ).

\section{Sample}

On the Numerical Rating Scale, 111 patients (40.2\%) reported a subjective treatment urgency between 1 point and 5 points (defined as low urgency) and 165 patients $(59.8 \%)$ reported between 6 points and 10 points (defined as high urgency). In the final assessment of the medical and nursing staff of the hospitals, 17 patients $(7.1 \%)$ were triaged as 'non-urgent', 144 patients (60.3\%) as 'standard' and 78 patients $(32.6 \%)$ as 'urgent'. A lower subjective treatment urgency was significantly associated with a higher (ie, less urgent) triaging by the hospital staff $(-0.17,95 \%$ CI -0.28 to $0.06, p=0.002)$. The interrelation between both variables is shown in figure 3 .

The patient population is described in table 1 . The patients had a mean age of 50.1 years. Patients with a low subjective treatment urgency were younger than patients with high subjective urgency (46.1 years vs 53.2 years, $\mathrm{p}=0.002)$. More than half of the patients $(51.8 \%)$ were women. The educational level was mostly medium $(59.6 \%)$ or low $(24.4 \%)$. Patients who rated their treatment urgency as low reported less impairment in quality of life $(0.60$ vs 0.45 in relation to 1 for full health, $\mathrm{p}=0.002)$ and less somatic symptoms (6.3 vs 8.3 on the $0-30$ points scale, $\mathrm{p}=0.008$ ) than patients rating their treatment urgency as high.

Almost half of the patients received medical diagnoses from the musculoskeletal system (44.1\%). Other frequently affected organ systems were the skin $(26.3 \%)$, 'general and unspecified disorders' $(17.8 \%)$, the neurological system $(8.0 \%)$, the digestive system $(6.6 \%)$, the respiratory system $(5.6 \%)$ and the cardiovascular system $(5.6 \%)$. Patients with low subjective treatment urgency received more diagnoses from the musculoskeletal system (53.2\% vs $37.0 \%, \mathrm{p}=0.018)$ and less diagnoses from the digestive system $(2.1 \%$ vs $10.1 \%, \mathrm{p}=0.020)$ than patients with high subjective treatment urgency.

The most frequently used diagnosis type was 'injuries' $(44.1 \%)$, followed by 'symptoms/complaints' $(36.2 \%)$, 'other diagnoses' $(35.7 \%)$, 'infections' $(9.4 \%)$ and 'neoplasms' $(0.9 \%)$. Patients rating their treatment urgency as low more frequently received the diagnosis 


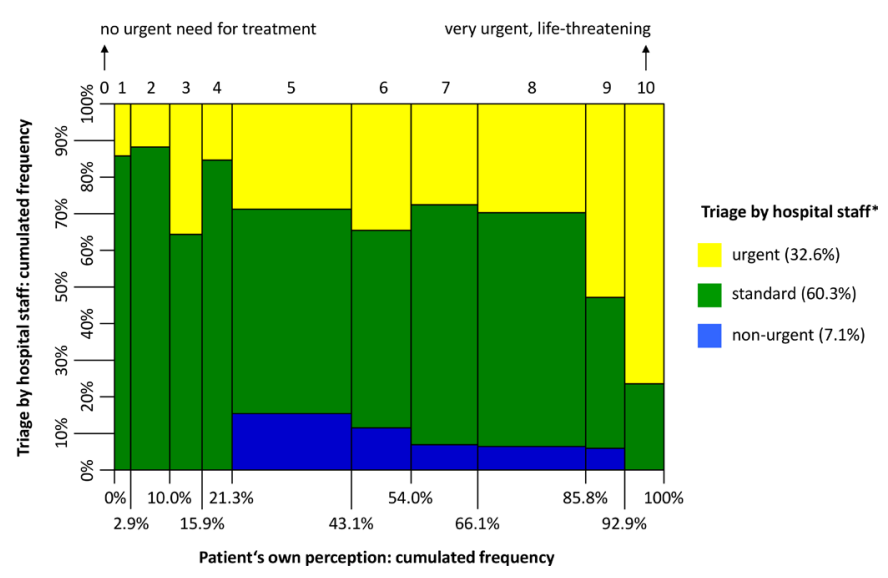

Figure 3 Treatment urgency rated by hospital staff and patient $(n=239)$. *Patients with immediate or very urgent need of treatment have been excluded.

type 'injuries' ( $53.2 \%$ vs $37.0 \%, \mathrm{p}=0.018$ ) than patients who rated their treatment urgency as high.

\section{Analysis of subjective treatment urgency}

The association of subjective treatment urgency with sociodemographic data, health status, health literacy and commitment to the GP is presented in table 2. In model 1 , a lower age and male sex were associated with a lower subjective treatment urgency. However, this association disappeared after adjusting for health status in model 2 and the model fit improved significantly $(p<0.001)$. Instead, less impairment in health-related quality of life, less somatic symptoms and a higher level of anxiousness were associated with a lower subjective treatment urgency. These factors remained statistically significant after including health literacy and commitment to the GP in model 3 and the model fit did not further improve $(\mathrm{p}=0.093)$. Furthermore, in model 3, there was an association of male sex and a lower intensity of commitment to the GP with a lower subjective treatment urgency, but we did not find an association between health literacy and the subjective treatment urgency.

\section{DISCUSSION}

\section{Statement of principal findings}

Our study analysed if subjectively perceived treatment urgency is associated with self-reported health literacy and the willingness to use the GP as coordinator of treatment. In our final model, a lower level of subjectively perceived treatment urgency was predicted by male sex, less impairment in quality of life and less somatic symptoms, but a higher level of anxiousness. Furthermore, a lower willingness to use the GP as coordinator of treatment also predicted a lower perceived treatment urgency. The question if patients had a regular GP and the selfreported health literacy did not predict the patients' perceived treatment urgency in our study.

\section{Comparison with the literature}

The subjective treatment urgency of patients in German emergency departments had already been examinedusing different predictor variables-in our preceding study 'PiNo Nord'. ${ }^{7}$ In this study, the proportion of patients rating their treatment urgency as 'low' was higher than in our recent study 'PiNo Bund' ( $54.7 \%$ vs $40.2 \%$ ) and there was little correlation between triage level and self-rated treatment urgency. The reduced number of low urgency visits and the stronger association between triage level and subjective treatment urgency in PiNo Bund could be an effect of various measures for improving patient allocation being implemented in Germany's emergency care after the PiNo Nord results had been published, for example, establishing a higher number of outpatient emergency practices located in hospitals, ${ }^{25}$ redesigning the emergency medical services of the Associations of Statutory Health Insurance Physicians ${ }^{26}$ and implementing a structured medical first assessment in their telephone counselling services. ${ }^{27}$ However, differences might also be explained by differences in the location and care situation of the study hospitals between both studies. PiNo Bund focused on small cities and rural regions with a medium to very high ACS density. In contrast, four of the five study hospitals in PiNo Nord were located in large university cities, rural regions were not represented and all administration districts in PiNo Nord had a low ACS density.

In our PiNo Bund study, we saw associations of younger age with a lower subjective urgency rating, which disappeared after adjusting for health status. In a systematic review, six out of nine identified studies found an association between younger age and a higher probability of non-urgent emergency department use. ${ }^{10}$ In line with these findings, in a recent German study, a higher age was related to a lower likeliness of self-referred walk-in visits at the emergency department. ${ }^{8}$

In two of three statistical models, we found an association between sex and subjective treatment urgency. If only sociodemographic data were included in the statistical model, male sex predicted a lower urgency. After adjusting for health status, health literacy and commitment to the GP, male sex again was associated with lower urgency. Other studies found inconsistent results regarding the influence of sex. For example, in the systematic review described above, four studies suggested a higher likelihood of non-urgent visits of women, two studies suggested a higher probability in men and four studies found no relationship between sex and nonurgent emergency department utilisation..$^{10}$

In patients with lower treatment urgency, we saw less impairment in quality of life and a lower burden of somatic symptoms than in patients with higher subjective treatment urgency. A systematic review found mixed evidence regarding the association between health status and utilisation of emergency departments for non-urgent conditions. Two studies saw a higher utilisation of patients with bad health condition and two studies did not find 
Table 1 Patient population by subjective treatment urgency

\begin{tabular}{|c|c|c|c|c|}
\hline & $\begin{array}{l}\text { Total } \\
(n=276)\end{array}$ & $\begin{array}{l}\text { Low subjective } \\
\text { treatment urgency } \\
(\mathrm{n}=111)\end{array}$ & $\begin{array}{l}\text { High subjective } \\
\text { treatment urgency } \\
(n=165)\end{array}$ & $P$ value \\
\hline $\begin{array}{l}\text { Age } \\
\text { (in years) }\end{array}$ & $\begin{array}{l}50.1 \pm 18.8 \\
(n=275)\end{array}$ & $\begin{array}{l}\text { 46.1 } 17.1 \\
(n=110)\end{array}$ & $\begin{array}{l}53.2 \pm 19.3 \\
(n=165)\end{array}$ & 0.002 \\
\hline \multicolumn{5}{|l|}{ Sex } \\
\hline Female & $51.8 \%$ & $48.7 \%$ & $53.9 \%$ & 0.388 \\
\hline \multicolumn{5}{|l|}{ Education pursuant to CASMIN } \\
\hline Low & $24.4 \%$ & $19.4 \%$ & $27.8 \%$ & \multirow[t]{3}{*}{0.191} \\
\hline Medium & $59.6 \%$ & $61.1 \%$ & $58.6 \%$ & \\
\hline High & $\begin{array}{l}15.9 \% \\
(n=270)\end{array}$ & $\begin{array}{l}19.4 \% \\
(n=108)\end{array}$ & $\begin{array}{l}13.6 \% \\
(n=162)\end{array}$ & \\
\hline $\begin{array}{l}\text { Somatic symptoms } \\
\text { (pursuant to } \mathrm{PHQ}-15 \text {, score } \\
\text { range from } 0 \text { point to } 30 \text { points) }\end{array}$ & $\begin{array}{l}7.4 \pm 5.0 \\
(n=170)\end{array}$ & $\begin{array}{l}6.3 \pm 4.7 \\
(n=77)\end{array}$ & $\begin{array}{l}8.3 \pm 5.1 \\
(n=93)\end{array}$ & 0.008 \\
\hline $\begin{array}{l}\text { Depressiveness (pursuant to } \\
\text { HADS subscale depression, } \\
\text { score range from } 0 \text { point to } 21 \\
\text { points) }\end{array}$ & $\begin{array}{l}3.9 \pm 3.8 \\
(n=248)\end{array}$ & $\begin{array}{l}3.7 \pm 3.2 \\
(n=97)\end{array}$ & $\begin{array}{l}4.1 \pm 4.2 \\
(n=151)\end{array}$ & 0.401 \\
\hline $\begin{array}{l}\text { Anxiousness (pursuant to } \\
\text { HADS subscale anxiety, score } \\
\text { range from } 0 \text { point to } 21 \text { points) }\end{array}$ & $\begin{array}{l}5.3 \pm 4.1 \\
(n=249)\end{array}$ & $\begin{array}{l}5.3 \pm 4.1 \\
(n=97)\end{array}$ & $\begin{array}{l}5.3 \pm 4.1 \\
(n=152)\end{array}$ & 0.989 \\
\hline
\end{tabular}

Statistically significant results $(p \leq 0.05)$ are shown in bold and italic. Low subjective treatment urgency: between 0 point and 5 points; high subjective treatment urgency: between 6 points and 10 points.

CASMIN, Comparative Analysis of Social Mobility in Industrial Nations; EQ-5D, EuroQol Five-Dimension Scale; F-HaBi, Questionnaire on Intensity of the Commitment to the GP ('Fragebogen zur Intensität der Hausarztbindung'); GP, general practitioner; HADS, Hospital Anxiety and Depression Scale; HLS-EU-Q16, European Health Literacy Questionnaire, 16 questions version; PHQ-15, Patient Health Questionnaire, 15 items version.

an association between those two factors. ${ }^{10}$ A German study found that patients with somatoform disorders more frequently attended out-of-hours care than patients without somatisations. ${ }^{28}$

In our study, a higher level of anxiousness was another significant predictor of lower subjective treatment urgency. A qualitative study from Germany mentioned health anxiety as an essential motive of patients visiting the emergency department for conditions with low treatment urgency. ${ }^{9}$ Three American studies highlighted an increased prevalence of anxiety disorders among frequent visitors of the emergency department. ${ }^{29-31}$ Fear and uncertainty have been identified as important motives of patients with chronic conditions to use emergency care. ${ }^{32}$ In contrast, a systematic review found no significant effect of anxiety disorders on the use of urgent care in the eight identified studies. ${ }^{33}$

We did not find a relationship between depressiveness and self-reported treatment urgency in our study. A systematic review showed mixed evidence regarding the effect of depression. Of the 16 included studies, only 8 showed a significant effect of depression on the utilisation of urgent healthcare. ${ }^{34}$ More recently, two other studies found an association between depression and emergency department utilisation. ${ }^{35} 36$ 
Table 2 The association of subjective treatment urgency with sociodemographic data, health status, health literacy and commitment to the GP: results of multivariable linear regression analyses adjusted for random effects on the hospital level

\begin{tabular}{|c|c|c|c|c|c|c|}
\hline & \multicolumn{2}{|l|}{ Model 1} & \multicolumn{2}{|l|}{ Model 2} & \multicolumn{2}{|l|}{ Model 3} \\
\hline & $\beta(95 \% \mathrm{Cl})$ & $P$ value & $\beta(95 \% \mathrm{Cl})$ & $P$ value & $\beta(95 \% \mathrm{Cl})$ & $P$ value \\
\hline Age (per 10 years) & 0.31 (0.09 to 0.53$)$ & 0.005 & $0.18(-0.02$ to 0.38$)$ & 0.082 & $0.18(-0.03$ to 0.38$)$ & 0.091 \\
\hline Sex (women vs men) & $1.06(0.28$ to 1.85$)$ & 0.008 & $0.67(-0.06$ to 1.40$)$ & 0.074 & 0.84 (0.11 to 1.57$)$ & 0.024 \\
\hline \multicolumn{7}{|c|}{ Education (pursuant to CASMIN) } \\
\hline $\begin{array}{l}\text { Medium level vs } \\
\text { low level }\end{array}$ & $0.48(-1.48$ to 0.52$)$ & 0.320 & $0.39(-1.30$ to 0.52$)$ & 0.404 & $0.27(-1.17$ to 0.64$)$ & 0.560 \\
\hline $\begin{array}{l}\text { High level vs low } \\
\text { level }\end{array}$ & $1.00(-2.19$ to 0.19$)$ & 0.100 & $0.49(-1.59$ to 0.60$)$ & 0.378 & $0.56(-1.65$ to 0.52$)$ & 0.309 \\
\hline $\begin{array}{l}\text { Health-related quality } \\
\text { of life } \\
\text { (pursuant to EQ-5D, } \\
\text { value set UK) }\end{array}$ & & & $-2.42(-3.55$ to -1.28$)$ & $<0.001$ & $-2.27(-3.39$ to -1.15$)$ & $<0.001$ \\
\hline $\begin{array}{l}\text { Somatic symptoms } \\
\text { (pursuant to PHQ-15) }\end{array}$ & & & $0.08(0.002$ to 0.17$)$ & 0.044 & $0.09(0.004$ to 0.17$)$ & 0.040 \\
\hline $\begin{array}{l}\text { Depressiveness } \\
\text { (pursuant to HADS } \\
\text { subscale depression) }\end{array}$ & & & $0.07(-0.05$ to 0.19$)$ & 0.258 & $0.09(-0.03$ to 0.21$)$ & 0.159 \\
\hline $\begin{array}{l}\text { Anxiousness } \\
\text { (pursuant to HADS } \\
\text { subscale anxiety) }\end{array}$ & & & $-0.13(-0.25$ to -0.02$)$ & 0.021 & $-0.13(-0.24$ to -0.01$)$ & 0.027 \\
\hline $\begin{array}{l}\text { Health literacy } \\
\text { (pursuant to HLS- } \\
\text { Q16-EU) }\end{array}$ & & & & & $0.08(-0.04$ to 0.19$)$ & 0.214 \\
\hline $\begin{array}{l}\text { Patient has an } \\
\text { attending GP }\end{array}$ & & & & & $-0.61(-2.46$ to 1.23$)$ & 0.514 \\
\hline $\begin{array}{l}\text { Intensity of } \\
\text { commitment to the } \\
\text { GP } \\
\text { (pursuant to F-HaBi) }\end{array}$ & & & & & $0.08(0.01$ to 0.14$)$ & 0.029 \\
\hline
\end{tabular}

Statistically significant results $(\mathrm{p} \leq 0.05)$ are shown in bold and italic; $95 \% \mathrm{Cl}$ : $95 \%$ confidence interval.

CASMIN, Comparative Analysis of Social Mobility in Industrial Nations; EQ-5D, EuroQol Five-Dimension Scale; F-HaBi, Questionnaire on Intensity of the Commitment to the GP ('Fragebogen zur Intensität der Hausarztbindung'); GP, general practitioner; HADS, Hospital Anxiety and Depression Scale; HLS-EU-Q16, European Health Literacy Questionnaire, 16 questions version; PHQ-15, Patient Health Questionnaire, 15 items version.

In our study, health literacy was not related to subjective treatment urgency. In the literature, there is mixed evidence about this relationship. For example, two studies from the USA found that inadequate health literacy was directly related to a higher probability of emergency department visits $^{37}$ and higher emergency department costs in elderly patients. ${ }^{38}$ Another American study also found a higher likeliness of emergency department visits of patients with low health literacy but no association between health literacy and accessing outpatient healthcare.$^{39}$ In contrast, a recent study from Australia found that a higher health literacy improved the understanding of the health problem of patients with low treatment urgency but did not lead to a better utilisation of healthcare resources. ${ }^{40}$ Another recent study from the USA also found no relationship between health literacy and emergency department utilisation. ${ }^{41}$

We did not find an association between having a regular GP and the self-reported treatment urgency, but the willingness to use the GP as coordinator of treatment predicted a higher subjective treatment urgency. The subjectively perceived lack of availability of primary care is a frequently mentioned reason for attending emergency departments with conditions of low treatment urgency. ${ }^{710}$ In many international studies, lack of access to primary care predicted a higher inappropriate use of emergency care. ${ }^{42-44}$ In a recent German study, having a regular GP was associated with a lower probability of self-referred walk-in consultations at the emergency department. ${ }^{8}$ In contrast, a German qualitative study concluded that primary care utilisation patterns and GP-patient relationship had limited relevance for the decision to use emergency departments. ${ }^{45}$

Implications for clinical practice

Our study focused on factors associated with the subjective treatment urgency, which is not identical with the triage level rated by the medical and nursing staff of the 
hospitals. However, there is a strong statistically significant association between both variables suggesting that results might be comparable if a clinical rating of treatment urgency was used instead of the patient rating.

A factor associated with a low subjective urgency is a high level of anxiousness. Anxious patients who assess their treatment urgency as low, but still want to make sure that their health problem is not dangerous, might benefit from a better advertising of the not very well-known ${ }^{7}$ telephone counselling services of the of the Associations of Statutory Health Insurance Physicians. Another possible response could be better patient education concerning the treatment of chronic health problems like diabetes, which might be connected with high levels of anxiety. ${ }^{46}$ Also, the identification and treatment of anxiety disorders in primary care should be improved by a better implementation of available screening instruments and management strategies. ${ }^{47}$

The results of our study suggest that the commitment to the GP and the willingness to use him or her as coordinator of treatment is associated with subjective treatment urgency. Strengthening the patients' GP commitment might, therefore, help reducing visits to emergency departments of patients with low treatment urgency. GP commitment might be improved by various strategies. In Germany, special health insurance tariffs like 'Hausarztzentrierte Versorgung' ('GP-centred care', based on the obligation to always consult the GP first in the event of health problems) exist, but they are unequally distributed across the German regions. For example, more than $80 \%$ of the patients using GP-centred care are located in the federal states of Bavaria and Baden-Württemberg. ${ }^{48}$ Promotion in the other 14 federal states might significantly increase the number of patients using these tariffs.

Another possibility would be a comprehensive media campaign. One example for such a campaign is the 'psychenet' study, which used different media like placards, cinema ads, classified ads, radio ads, brochures and a website to improve awareness of mental disorders in the population. ${ }^{49}$ A similar strategy could be used to educate patients about the importance of GP coordination. In this campaign, it should be considered that GP commitment is associated with different sociodemographic data. ${ }^{11}$ The media campaign could, therefore, include targeted interventions for groups with low GP commitment like women or patients from regions with higher urban density, ${ }^{11}$ which could receive specific information about why they in particular would profit from GP coordination.

\section{Strengths and limitations}

One strength of our study is the careful selection of included hospitals based on an analysis of the outpatient and inpatient care situation throughout Germany. ${ }^{13}$ In the design of the study, we also could build on our experiences with a similar study of 1299 emergency department patients ${ }^{7}$ and we adapted the procedures of patient recruitment and data collection to the local situation in consensus with the respective hospitals. The personnel was thoroughly trained and monitored. The analysed constructs were operationalised by established and validated questionnaires. ${ }^{16-22}$ And the statistical methods provided additional strength by considering potential confounders and the cluster structure of the dataset.

The assessment of the commitment to the GP was based on the $\mathrm{F}-\mathrm{HaBi}$, for which one-dimensional factor structure, adequate high internal consistency and satisfactory item-total correlation of all items have been demonstrated in another paper. ${ }^{11}$ The F-HaBi is used in a number of other studies, ${ }^{11}{ }^{50-53}$ but due to the fact that the F-HaBi is a relatively new questionnaire, as yet there is only one with published results. ${ }^{11}$ For this reason, at the present time, there is little possibility to compare our results with other settings or populations.

Factors limiting the representativeness of the study arise from the selection of districts, patient exclusion and recourse. We did not recruit hospitals from districts with a low rate of ACS. The study also only includes districts in eastern, central and western Germany, but no districts in northern or southern Germany. Furthermore, four out of the five study hospitals were located in rural regions and only one urbanised district is covered. Large metropolitan areas like Hamburg or Berlin are not represented in the study.

Patients were excluded from the study if they needed treatment immediately or very urgently, or if they had severe symptoms. Furthermore, patients were excluded if they had received treatment-due to low patient numbers at certain times of the day-before receiving information, giving consent, being interviewed or if they were directly referred to a different department within the hospital. The latter particularly applied to female patients consulting for gynaecological symptoms. Furthermore, the data collection took place during day shifts only.

We had a low response rate of $41.2 \%$ mainly resulting from $40.1 \%$ of eligible patients refusing participation in our study. In our non-responder analysis, study participation was independent of age and sex, but we had no other data on non-responders. However, there is evidence from other studies that specific groups, for example, patients with the lowest socioeconomic position ${ }^{54} 55$ or health conditions connected to social desirability like current smoking, heavy alcohol use, panic disorder and use of tranquillisers, ${ }^{55}$ are less likely to participate in studies. Therefore, it might be that our data are not representative for these groups.

As in most surveys, recall problems, errors and social desirability are possible sources of bias. To minimise these effects, the interviewers received multiple training sessions before the interviews started and were supervised throughout the entire survey period. As PiNo Bund was an observational study with multiple target variables, it was not possible to carry out a sample size calculation. Instead, the sample size was determined based on our experience with similar studies. It is, therefore, possible that we missed significant predictors of our target variable due to limited statistical power. 


\section{CONCLUSIONS}

In our study, patients with less willingness to use GPs as coordinators of the treatment were more likely to visit emergency departments with health problems that are perceived by themselves as non-urgent. Effective strategies for strengthening the patients' GP commitment might, therefore, be helpful for reducing emergency department crowding and its adverse effects on patients and the hospital staff.

\section{Author affiliations}

${ }^{1}$ Department of Primary Medical Care, University Medical Center HamburgEppendorf, Hamburg, Germany

${ }^{2}$ Department of Emergency and Acute Medicine, Charite Universitatsmedizin Berlin, Berlin, Germany

\section{Twitter Anna Slagman @annaSlagman and Martin Scherer @degampraesident}

Acknowledgements We would like to express our appreciation to the five hospitals who facilitated this study with their participation.

Contributors IS, AM, JHO, DL and MS conceived and designed the study. IS performed the statistical analyses and drafted the manuscript. AS and MM contributed to the discussion of the study results. All the authors commented on the draft and read and approved the final version of the manuscript. MS is the guarantor.

Funding The study was funded by Central Research Institute of Ambulatory Health Care in Germany (no grant number received). The funding body provided results from an analysis used for recruitment of the participating hospitals, but otherwise had no role in the design of the study and collection, analysis and interpretation of data and in writing the manuscript.

Map disclaimer The inclusion of any map (including the depiction of any boundaries therein), or of any geographic or locational reference, does not imply the expression of any opinion whatsoever on the part of BMJ concerning the legal status of any country, territory, jurisdiction or area or of its authorities. Any such expression remains solely that of the relevant source and is not endorsed by BMJ. Maps are provided without any warranty of any kind, either express or implied.

Competing interests None declared.

Patient and public involvement Patients and/or the public were not involved in the design, or conduct, or reporting, or dissemination plans of this research.

\section{Patient consent for publication Not applicable.}

Ethics approval The study was approved by the Ethics Committee of the Hamburg Medical Association on 22 July 2015 and amended on 7 March 2017 and 30 December 2019 (approval number: PV4993).

Provenance and peer review Not commissioned; externally peer reviewed.

Data availability statement No data are available. The ethics approval does not allow data sharing.

Open access This is an open access article distributed in accordance with the Creative Commons Attribution Non Commercial (CC BY-NC 4.0) license, which permits others to distribute, remix, adapt, build upon this work non-commercially, and license their derivative works on different terms, provided the original work is properly cited, appropriate credit is given, any changes made indicated, and the use is non-commercial. See: http://creativecommons.org/licenses/by-nc/4.0/.

\section{ORCID iDs}

Ingmar Schäfer http://orcid.org/0000-0002-1038-7478

Martin Möckel http://orcid.org/0000-0002-7691-3709

\section{REFERENCES}

1 Lowthian JA, Curtis AJ, Jolley DJ, et al. Demand at the emergency department front door: 10-year trends in presentations. Med J Aust 2012;196:128-32.

2 Somasundaram R, Geissler A, Leidel BA. Beweggründe für die Inanspruchnahme von Notaufnahmen - Ergebnisse einer
Patientenbefragung [Reasons for Emergency Department Visits: Results of a Patient Survey]. Gesundheitswesen 2018;80:621-7.

3 Morley C, Unwin M, Peterson GM, et al. Emergency department crowding: a systematic review of causes, consequences and solutions. PLoS One 2018;13:e0203316.

4 Carter EJ, Pouch SM, Larson EL. The relationship between emergency department crowding and patient outcomes: a systematic review. J Nurs Scholarsh 2014;46:106-15.

5 Chiu I-M, Lin Y-R, Syue Y-J, et al. The influence of crowding on clinical practice in the emergency department. Am J Emerg Med 2018;36:56-60.

6 Behringer W, Buergi U, Christ M. Fünf Thesen zur Weiterentwicklung der Notfallmedizin in Deutschland, Österreich und der Schweiz. [Five theses on the further development of emergency medicine in Germany, Austria and Switzerland. Notfall Rettungsmed 2013;16:625-6.

7 Scherer M, Lühmann D, Kazek A. Patients attending emergency departments. A cross-sectional study of subjectively perceived treatment urgency and motivation for attending. Dtsch Arztebl Int 2017;114:645-52.

8 Holzinger F, Oslislo S, Möckel M, et al. Self-referred walk-in patients in the emergency department - who and why? Consultation determinants in a multicenter study of respiratory patients in Berlin, Germany. BMC Health Serv Res 2020;20:848.

9 Schmiedhofer M, Möckel M, Slagman A, et al. Patient motives behind low-acuity visits to the emergency department in Germany: a qualitative study comparing urban and rural sites. BMJ Open 2016;6:e013323.

10 Uscher-Pines L, Pines J, Kellermann A. Deciding to visit the emergency department for non-urgent conditions: a systematic review of the literature. Am J Manag Care 2013;19:47-59.

11 Hansen H, Schäfer I, Porzelt S, et al. Regional and patient-related factors influencing the willingness to use general practitioners as coordinators of the treatment in northern Germany - results of a cross-sectional observational study. BMC Fam Pract 2020;21:110.

12 Sørensen K, Van den Broucke S, Fullam J, et al. Health literacy and public health: a systematic review and integration of definitions and models. BMC Public Health 2012;12:80.

13 Erhart M, Rincón I, Özkan A. Auswahl von Standorten für die Multicenterstudie Patienten in Notaufnahme basierend auf einem regionalen „Theoretical“ Sampling. Identifikation regionaler Cluster stationärer Notaufnahme-,, Typen“. Berlin: Central Research Institute of Ambulatory Health Care in Germany, 2016.

14 Schuettig W, Sundmacher L. Ambulatory care-sensitive emergency department cases: a mixed methods approach to systemize and analyze cases in Germany. Eur J Public Health 2019;29:1024-30.

15 Bundesinstitut für Bau- Stadt- und Raumforschung. Siedlungsstrukturelle Kreistypen - Laufende Raumbeobachtung - Raumabgrenzungen. Available: http://www.bbsr.bund.de/nn 1067638/BBSR/DE/Raumbeobachtung/Raumabgrenzungen/ Kreistypen4/kreistypen.html [Accessed 17 Jan 2019].

16 WONCA International Classification Committee. ICPC-2-R: International classification of primary care. Revised 2nd ed. Oxford: Oxford University Press, 2005.

17 Azeredo TRM, Guedes HM, Rebelo de Almeida RA, et al. Efficacy of the Manchester triage system: a systematic review. Int Emerg Nurs 2015;23:47-52

18 Brauns H, Steinmann S. Educational reform in France, WestGermany and the United Kingdom: updating the CASMIN educational classification. ZUMA-Nachrichten 1999;44:7-44.

19 EuroQol Group. EuroQol - a new facility for the measurement of health-related quality of life. Health Policy 1990;16:199-208.

20 Szende A, Oppe M, Devlin N. EQ-5D value sets. Inventory, comparative review and user guide. Berlin: Springer Netherland, 2007.

21 Zigmond AS, Snaith RP. The hospital anxiety and depression scale. Acta Psychiatr Scand 1983;67:361-70.

22 Kroenke K, Spitzer RL, Williams JBW. The PHQ-15: validity of a new measure for evaluating the severity of somatic symptoms. Psychosom Med 2002;64:258-66.

23 Sørensen K, Pelikan JM, Röthlin F, et al. Health literacy in Europe: comparative results of the European health literacy survey (HLS-EU). Eur J Public Health 2015;25:1053-8.

24 Schäfer I, Scherer M, Lühmann D. In reply. Dtsch Arztebl Int 2018;115:66-7.

25 Kopetsch T, Steffen L. Ambulante Notfallbehandlung in der Gesetzlichen Krankenversicherung - Eine empirische analyse. ZfWP 2020;69:203-31.

26 Krefis AC, Fischereit J, Hoffmann P, et al. Temporal analysis of determinants for respiratory emergency department visits in a large German hospital. BMJ Open Respir Res 2018;5:e000338. 
27 Graf von Stillfried D, Czihal T, Meer A. State of the art reportstructured medical first assessment in Germany (SmED)]. Notfall Rettungsmed 2019;22:578-88.

28 Leutgeb R, Berger S, Szecsenyi J, et al. Patients with somatoform disorders: more frequent attendance and higher utilization in primary out-of-hours care? PLoS One 2018;13:e0202546.

29 Cunningham A, Mautner D, Ku B, et al. Frequent emergency department visitors are frequent primary care visitors and report unmet primary care needs. J Eval Clin Pract 2017;23:567-73.

30 Hardy M, Cho A, Stavig A, et al. Understanding frequent emergency department use among primary care patients. Popul Health Manag 2018;21:24-31.

31 McBride SM, Braz VA, Jones CW. Occult suicidality and psychiatric disease among emergency department patients with low-acuity chief complaints. West J Emerg Med 2018;19:573-8.

32 Rising KL, Hudgins A, Reigle M, et al. "I'm Just a Patient": Fear and Uncertainty as Drivers of Emergency Department Use in Patients With Chronic Disease. Ann Emerg Med 2016;68:536-43.

33 Blakeley C, Blakemore A, Hunter C, et al. Does anxiety predict the use of urgent care by people with long term conditions? A systematic review with meta-analysis. $J$ Psychosom Res 2014;77:232-9.

34 Dickens $\mathrm{C}$, Katon W, Blakemore A, et al. Does depression predict the use of urgent and unscheduled care by people with long term conditions? A systematic review with meta-analysis. J Psychosom Res 2012;73:334-42.

35 Guthrie EA, Dickens C, Blakemore A, et al. Depression predicts future emergency hospital admissions in primary care patients with chronic physical illness. J Psychosom Res 2016;82:54-61.

36 Blakemore A, Dickens C, Chew-Graham CA, et al. Depression predicts emergency care use in people with chronic obstructive pulmonary disease: a large cohort study in primary care. Int J Chron Obstruct Pulmon Dis 2019;14:1343-53.

37 Cho YI, Lee S-YD, Arozullah AM, et al. Effects of health literacy on health status and health service utilization amongst the elderly. Soc Sci Med 2008;66:1809-16.

38 Howard DH, Gazmararian J, Parker RM. The impact of low health literacy on the medical costs of Medicare managed care enrollees. Am J Med 2005;118:371-7.

39 Baker DW, Gazmararian JA, Williams MV, et al. Health literacy and use of outpatient physician services by Medicare managed care enrollees. J Gen Intern Med 2004;19:215-20.

40 Wayment A, Wong C, Byers S, et al. Beyond access block: understanding the role of health literacy and self-efficacy in lowacuity emergency department patients. Ochsner J 2020;20:161-9.

41 Caldwell EP. The influence of health literacy on emergency department utilization and hospitalizations in adolescents with sickle cell disease. Public Health Nurs 2019;36:765-71.

42 van den Berg MJ, van Loenen T, Westert GP. Accessible and continuous primary care may help reduce rates of emergency department use. An international survey in 34 countries. Fam Pract 2016;33:42-50.
43 Carret MLV, Fassa AG, Kawachi I. Demand for emergency health service: factors associated with inappropriate use. BMC Health Serv Res 2007;7:131.

44 Petersen LA, Burstin HR, O'Neil AC, et al. Nonurgent emergency department visits: the effect of having a regular doctor. Med Care 1998;36:1249-55.

45 Oslislo S, Heintze C, Möckel M, et al. What role does the GP play for emergency department utilizers? A qualitative exploration of respiratory patients' perspectives in Berlin, Germany. BMC Fam Pract 2020;21:154.

46 Collins MM, Corcoran P, Perry IJ. Anxiety and depression symptoms in patients with diabetes. Diabet Med 2009;26:153-61.

47 Combs H, Markman J. Anxiety disorders in primary care. Med Clin North Am 2014;98:1007-23.

48 Lübeck R, Beyer M, Gerlach F. Rationale und Stand der hausarztzentrierten Versorgung in Deutschland [,Rationale and dissemination of "GP centered health care" ("HzV") in Germany"]. Bundesgesundheitsblatt Gesundheitsforschung Gesundheitsschutz 2015;58:360-6.

49 Härter M, Kentgens M, Brandes A, et al. Rationale and content of psychenet: the Hamburg network for mental health. Eur Arch Psychiatry Clin Neurosci 2012;262:57-63.

50 Heddaeus D, Dirmaier J, Brettschneider C, et al. Study protocol for the comet study: a cluster-randomised, prospective, parallel-group, superiority trial to compare the effectiveness of a collaborative and stepped care model versus treatment as usual in patients with mental disorders in primary care. BMJ Open 2019;9:e032408.

51 Gemeinsamer Bundesausschuss. Innovationsausschuss. Förderbekanntmachungen. Förderprojekte. MULTlqual Entwicklung und Validierung von Qualitätsindikatoren für Multimorbidität. Available: https://innovationsfonds.g-ba.de/projekte/ versorgungsforschung/multiqual-entwicklung-und-validierung-vonqualitaetsindikatoren-fuer-multimorbiditaet.57 [Accessed 31 Aug 2021].

52 Gemeinsamer Bundesausschuss. Innovationsausschuss. Förderbekanntmachungen. Förderprojekte. demand Implementierung einer standardisierten Ersteinschätzung ALS basis eines demand managements in Der ambulanten Notfallversorgung. Available: https://innovationsfonds.g-ba.de/ projekte/versorgungsforschung/demand-implementierung-einerstandardisierten-ersteinschaetzung-als-basis-eines-demandmanagements-in-der-ambulanten-notfallversorgung.136 [Accessed 31 Aug 2021].

53 Jagodzinski A, Johansen C, Koch-Gromus U, et al. Rationale and design of the Hamburg City health study. Eur J Epidemiol 2020;35:169-81.

54 Korkeila K, Suominen S, Ahvenainen J, et al. Non-response and related factors in a nation-wide health survey. Eur $\mathrm{J}$ Epidemiol 2001;17:991-9.

55 Van Loon AJM, Tijhuis M, Picavet HSJ, et al. Survey non-response in the Netherlands: effects on prevalence estimates and associations. Ann Epidemiol 2003;13:105-10. 